\title{
The Blazhko Effect: Facts, Figures and Future work
}

\author{
K. Kolenberg \\ ${ }^{1}$ Institut für Astronomie, Türkenschanzstrasse 17, 1180 Vienna, Austria
}

\begin{abstract}
Almost a century after its discovery, the phenomenon of amplitude and/or phase modulation (Blazhko effect), observed in a large percentage of the RR Lyrae stars, still lacks widely acceptable theoretical understanding. Recent attempts to theoretically explain the Blazhko effect have focused on two alternatives: the magnetic models and the resonance models, both involving the presence of nonradial pulsation components. In the course of the past decades, large photometric data bases have yielded important statistics on the phenomenology of the Blazhko effect, which impose additional constraints upon the applicability of the models. On the other hand, the new approach of studying the Blazhko effect through line profile analysis, can provide essential information on the nature of the pulsation components responsible for the modulation. The answer to this century-old question will definitely clarify similar phenomena observed in other types of pulsating stars.
\end{abstract}

\section{Introduction: RR Lyrae stars and the Blazhko effect}

Since their discovery at the end of the $19^{\text {th }}$ century, the RR Lyrae stars have contributed to almost every branch of modern astronomy. These pulsating stars have mean periods of about half a day, and show brightness variations of about 1 magnitude. Traditionally, RR Lyrae stars are thought to represent the simplest type of pulsation, namely radial pulsation. They oscillate in the radial fundamental mode (Bailey type RRab), the radial first overtone (Bailey type RRc), and both of these radial modes simultaneously (RRd). However, additional cycles occur in many RR Lyrae stars. The most intriguing subclass of RR Lyrae stars consists of the stars showing the Blazhko effect. These stars have light curves that are modulated on timescales of tens to hundreds of days. Blazhko (1907) was the first to report this phenomenon in RW Dra. The estimated incidence rate of Blazhko variables among the Galactic RRab stars is about 20-30\% (Szeidl 1988; Moskalik \& Poretti 2003). In the LMC this rate is considerably lower (about 12\%; Alcock et al. 2003). RRc stars show the Blazhko effect even less frequently (less than 5\%). Characteristic for the phenomenon is the periodic variation of the brightness and times of maximum light of Blazhko stars over the secondary period (Figure 1a).

\section{Past and present observational studies of the Blazhko effect}

The Blazhko effect has been the frequent subject of photographic and photometric studies, e.g., the systematic investigations carried out at Konkoly Observatory in Budapest, Hungary (Szeidl \& Kollath 2000, and references therein). Traditionally the phenomenon was studied by means of $\mathrm{O}-\mathrm{C}$ analysis. Therefore the observations were in general strongly biased towards the ascending branch and maximum phase of the primary light curve, a sampling pernicious for Fourier analyses. Nevertheless, some photometric data sets, which also include other parts 


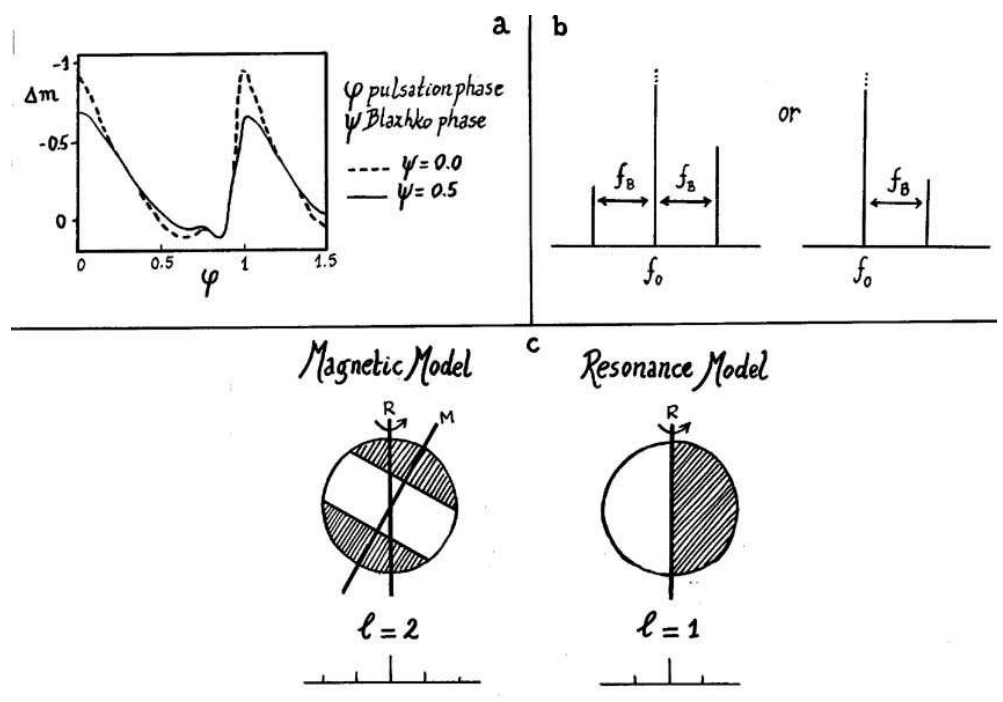

Figure 1: a. Light curve changes of a Blazhko star; b. Triplet or doublet structure; c. The two prevailing models for the Blazhko effect

of the light curve, allowed a rigorous frequency analysis,e.g., Borkowski (1980), Smith et al. (1994), Kovacs (1995), Nagy (1998), Smith et al. (1999), Szeidl \& Kollath (2000), Jurcsik et al. (2002a), Smith et al. (2003).

For a long time the presence of the Blazhko effect in RRc stars was an open question: because of the small changes in the height of maximum of their light curves it was more difficult to detect. The controversy was resolved by the systematic studies of accurate CCD data of globular clusters and the large variable star data bases resulting from microlensing surveys (MACHO for the LMC and OGLE for the Galactic Bulge). These studies have cast a new light upon the study of RR Lyrae variability (Moskalik \& Poretti 2003; Alcock et al. $2000,2003)$, and yielded important statistics on the phenomenology of the Blazhko effect.

The frequency spectra of light curves of RR Lyrae Blazhko stars exhibit either a doublet or an equally-spaced triplet structure, with a very small frequency separation close to the main radial pulsation component, corresponding to the frequency of the modulation (see Figure $1 b$ ). Only a small fraction of the observed triplets show non-equidistant frequency spacings. No trace of a quintuplet structure is found. The observed period ratios are 0.95-1.02. The modulation components have amplitudes of about $10-20 \%$ of the corresponding pulsation component and are also observed around the harmonics of the main frequency. If a triplet structure is observed, both side peaks appear to have unequal amplitudes, most often with the larger modulation peak at the higher frequency side of the main pulsation component. The continuous transition between the variables showing an equidistant triplet and those displaying only a close doublet suggests that both features are the result of the same phenomenon. No connection seems to exist between the pulsation and the Blazhko periods.

At their largest light amplitude Blazhko stars fall approximately on the curve of amplitude versus period as defined by stars with regular light curves (Szeidl 1988). Jurcsik et al. (2002b) tackled the important question what phase of the modulation, if any, can be regarded as undistorted behaviour. From studying photometric and radial velocity amplitudes of RRab Blazhko stars, as well as their light curve shapes, they concluded that for Blazhko stars with 
large amplitude modulation the light curves are always distorted.

Period changes, too fast to be of evolutionary nature, are a common feature in RR Lyrae stars, and also occur in Blazhko stars (Smith 1995; Szeidl \& Kollath 2000). Their origin is currently unknown. Coincident changes in the primary and Blazhko periods have been reported (e.g., LaCluzé et al. 2002). Some field Blazhko stars (e.g. RR Lyr, XZ Cyg, RV UMa, XZ Dra, RW Dra, Y LMi) are reported to display, besides their Blazhko cycles, also very long cycles, of the order of years. In some stars (SW And, RR Gem) the Blazhko effect ceased.

\section{Explanations for the Blazhko effect in RR Lyrae stars}

Almost a century after its discovery, the physical origin of the Blazhko effect remains a mystery. Different mechanisms have been proposed to explain the phenomenon, such as resonance effects, nonadiabatic effects, magnetic effects and tidal effects in binary systems. Models for the Blazhko effect using only radial mode interactions have failed (see Kovacs 2002). The most plausible hypotheses to explain this phenomenon focus on two types of models, both involving nonradial pulsation components.

- The resonance models are based on a (nonlinear) resonance between the radial fundamental mode and a nonradial mode. In these models the dipole modes $(\ell=1)$ have the highest probability to be nonlinearly excited (Cox 1993; Van Hoolst et al. 1998). The dipole pulsation components lead to a triplet structure in the frequency spectrum of Blazhko variables. In the model proposed by Nowakowski \& Dziembowski (2001), significant amplitude and phase modulation is predicted in the case of excitation of a rotationally split $\ell=1, m= \pm 1$ pair. The modulation period is determined by the rotation rate (currently unknown) and the Brunt-Väisälä frequency in the deepest part of the radiative interior. Peterson et al. (1996) obtained an upper limit of $10 \mathrm{~km} / \mathrm{s}$ for the projected rotational velocities of a sample of 27 RR Lyrae stars.

- The magnetic models, like the simple oblique pulsator model for roAp stars (Kurtz 1982), suppose that Blazhko stars have a magnetic field inclined to the stellar rotation axis (Cousens 1983; Shibahashi \& Takata 1995). The main radial mode is deformed by the magnetic field to have an additional quadrupole component $(\ell=2)$, for which the symmetry axis coincides with the magnetic axis. As the star rotates, the pulsation components change with observer aspect, causing the observed amplitude modulation. Shibahashi \& Takata (1995) predict a quintuplet structure in the frequency spectrum, but also show that the quintuplet looks almost like a triplet for certain geometrical configurations. Depending on which of the side components we then observe, the Blazhko period is supposed to be equal to the rotation period or half of the rotation period. A magnetic field of order $1 \mathrm{kG}$ is needed in this model for the amplitude modulation to be observable. Babcock (1958) and Romanov et al. (1994) reported a detection of a variable magnetic field in the brightest Blazhko star RR Lyrae with a strength up to $1.5 \mathrm{kG}$, but so far, these observations have not been confirmed (e.g., Preston 1967; Chadid 2001). A straightforward detection of magnetic fields in Blazhko stars is hampered by their relative faintness.

In both models the observed modulation of the light curve is a consequence of rotation and the degree of modulation is aspect-dependent. Each of the models predicts constant modulation components of equal amplitudes, in sharp contrast to the large majority of the frequency patterns observed. The question why RRc Blazhko stars have significantly lower incidence rates has not yet been addressed in the magnetic model. The resonance model does predict a lower probability for first overtone pulsators to show amplitude modulation, though still not in the degree observed. Deviations from strict amplitude/phase modulations (see e.g., 
Szeidl 1988) also need to be explained. The different incidence rates in different populations, probably related to metallicity (Moskalik \& Poretti 2003), have yet to be taken into account. Moreover, explanations predicting an undistorted light curve along any phase of a large amplitude modulation seem unrealistic (Jurcsik et al. 2002b). Empirical evidence suggests that RR Lyrae Blazhko stars have pulsation periods shorter than 0.66 days. This might be related to the fact that, when moving from the blue to the red edge of the instability strip, the convective transport in RR Lyrae stars becomes more efficient. Convective turbulence may play a role in driving/quenching the Blazhko effect (Bono et al. 2003).

\section{New developments in line profile analysis}

Up to now most observational studies of Blazhko stars were based on photometric data. However, high-resolution line profiles offer much better diagnostics to find and identify nonradial oscillation components in pulsating stars. The pioneering work in the spectroscopic study of the Blazhko effect consisted in studying the line profile variations of RR Lyrae, by far the brightest Blazhko star (Chadid et al. 1999; Kolenberg 2002). A detailed study of the variations of the Fell line profile at $4923.921 \AA$ led to a clear detection of nonradial pulsation components in the star and provided promising results concerning their identification. By means of an adapted version of the moment method, a quantitative spectroscopic identification method (Aerts 1996), the additional components were identified as non-axisymmetric nonradial dipole $(\ell=1)$ or quadrupole $(\ell=2)$ modes (Kolenberg 2002). Subsequent line profile fitting on the observed profiles confirmed these results. However, as the existing data did not provide a complete coverage of the Blazhko cycle, these results need to be checked on new data with a better spread over the Blazhko cycle. Similar high-resolution spectra of a non-Blazhko RR Lyrae star would allow us to carry out a spectroscopic comparison between Blazhko and non-Blazhko stars (see also Kolenberg et al. 2003).

\section{Long-term cycles in other types of stars}

The Blazhko effect is rather uncommon among the fundamental mode Cepheids. One of the few field Cepheids known to show strong amplitude modulation is HR 7308 (Breger 1981; Burki et al. 1982). Van Hoolst \& Waelkens (1995) considered the beating hypothesis for this star in a nonlinear framework and concluded that a resonance between the radial mode and a low-degree, low-order p-mode with nearly equal frequencies can produce periodic amplitude variations of the radial mode. Among multimode pulsators, amplitude and/or phase modulation is a rather common phenomenon. Recently Poretti (2003) showed that the pulsational content of the $\delta$ Scuti star V974 Oph suggests a mixture of radial and nonradial modes. The ratio $\mathrm{f} 1 / \mathrm{f} 2=0.978$ is reminiscent of the Blazhko effect in RR Lyrae stars. In FG Vir Breger et al. (2004) detected a nonradial mode very close to the radial mode. Finally, the $\beta$ Cephei star $16 \mathrm{Lac}$ also shows a radial and a close nonradial mode leading to amplitude variations (Aerts et al. 2003; Sareyan et al. 1997). These are just some examples illustrating the widespread occurrence of amplitude and/or phase modulation, i.e. Blazhkolike behaviour. A better understanding of the phenomenon in RR Lyrae stars will definitely clarify similar phenomena in other types of pulsating stars.

\section{The Blazhko project}

Despite the progress made in the past decades both in observations and modelling, the basic physical understanding of the Blazhko phenomenon is still missing. In order to join the efforts to come to a better understanding, we have set up an international collaboration centered on the Blazhko effect (the Blazhko project). The concept is to obtain sufficient high-resolution spectroscopic data of some well-selected field RR Lyrae Blazhko stars: both fundamental mode (RRab) stars and first harmonic (RRc) pulsators. Similar data sets for at least two non-modulated RR Lyrae stars (RRab and RRc) will permit a spectroscopic 
comparison of line profile variations between Blazhko and non-Blazhko stars. Moreover, they are essential as a test for the new methods to disentangle the (supposed nonradial) pulsation components which are suspected to be responsible for the amplitude modulation. Extensive photometric data and radial velocity measurements will be obtained to supplement the highresolution spectroscopic data and for accurate period determination. Target stars are carefully selected in both hemispheres. This new and so far untapped approach of combining highresolution spectra, additional radial velocity data, and extensive photometry of both Blazhko and non-Blazhko stars will provide a deeper insight in the mechanism behind the phenomenon. People who are interested in actively collaborating, either from the observational side (by collaborating in a Blazhko star campaign), or from the theoretical side (by participating in the interpretation of the measurements) are welcome to contact kolenberg@astro.univie.ac.at.

Acknowledgments. KK cordially thanks the SOC for inviting this contribution, for registration and accommodation grant. The LOC is acknowledged for their hospitality and smooth organisation. KK thanks Michel Breger for his encouraging support of this project, and all the people who have expressed their interest in the Blazhko effect and thereby motivated a larger-scale tackling of the problem.

\section{References}

Aerts, C., 1996, A\&A 314, 115

Aerts, C., Lehmann, H., Briquet, M., et al. 2003, A\&A 399, 639

Alcock, C., Allsmann, R., Alves, D.R., et al., 2003, in preparation

Alcock, C., Allsmann, R., Alves, D.R., et al., 2000, ApJ 542, 257

Babcock, H.W., 1958, ApJS 3, 141

Blazhko, S., 1907, Astron. Nachr. 175, 325

Bono, G., Petroni, S., Marconi, M., 2003, ASP Conf. Ser. 292, 71

Breger, M., 1981, ApJ 249, 666

Breger, M., Rodler, F., Pretorius, R., et al., 2004, A\&A, in press

Borkowski, K.J., 1980, Acta Astr. 30, 393

Burki, G., Mayor, M., Benz, W., 1982, A\&A 109, 258

Chadid, M., Kolenberg, K., Aerts, C, Gillet, D., 1999, A\&A 352, 201

Chadid, M., 2001, ASP Conf. Ser. 248, 427

Cousens, A., 1983, MNRAS 203, 1171

Cox, A.N., 1993, Proc. IAU Coll. 21, 31

Jurcsik, J., Benkö, J.M., \& Szeidl, B., 2002a, A\&A 396, 539

Jurcsik, J., Benkö, J.M., \& Szeidl, B., 2002b, A\&A 390, 133

Kolenberg, K., 2002, PhD thesis, http://www.ster.kuleuven.ac.be/pub/kolenberg_phd/

Kolenberg, K., Aerts, C., Chadid, M., Gillet, D., 2003, ASP Conf. Ser. 305, 167

Kovács, G., 1995, A\&A 295, 693

Kovács, G., 2002, ASP Conf. Ser. 259, 396

Kurtz, D.W., 1982, MNRAS 200, 807

LaCluyzé, A., Smith, H.A., Gil, E.-M. et al., 2002, ASP Conf. Ser. 259, 416

Moskalik, P., \& Poretti, E., 2003, A\&A 398, 213

Nagy, A., 1998, A\&A 339, 440

Nowakowski, R.M., \& Dziembowski, W.A., 2001, Acta Astron. 51, 5

Peterson, R.C., Carney, B.W., \& Latham, D.W., 1996, ApJ 465, 47

Poretti, E., 2003, A\&A 409, 1031

Preston, G.W., 1967, in The Magnetic and Related Stars, Baltimore Mono Book Corp., 3

Romanov, Yu.S., Udovichenko, S.N., Frolov, M.S., 1994, Bul. Spec. Astrophys. Obs. 38, 169

Sareyan, J.P., Chauville, J., Chapellier, E., Alvarez, M., 1997, A\&A 321, 145

Shibahashi, H., \& Takata, M., 1995, ASP Conf. Ser. 83, 42

Smith, H.A., Matthews, J.M., Lee, K.M., et al., 1994, ApJ 107, 679

Smith, H.A., 1995, RR Lyrae Stars, Cambridge Univ. Press

Smith, H.A., Barnett, M., silbermann, N.A., Gay, P., 1999, AJ 118, 572

Smith, H.A., Church, J.A., Fournier, J., et al. 2003, PASP 115, 43

Szeidl, B., 1988, in Multimode Stellar Pulsations, Proc. Budapest Workshop 1987, 45

Szeidl, B., \& Kolláth, Z., 2000, ASP Conf. Ser. 203, 281

Van Hoolst, T., \& Waelkens, C., 1995, A\&A 295, 361

Van Hoolst, T., Dziembowski, W.A., Kawaler, S.D., 1998, MNRAS 297, 536 